\title{
ORIGINAL ARTICLE \\ Basal metabolic rate can evolve independently of morphological and behavioural traits
}

\begin{abstract}
KJ Mathot ${ }^{1}$, K Martin ${ }^{2}$, B Kempenaers ${ }^{2}$ and W Forstmeier ${ }^{2}$
Quantitative genetic analyses of basal metabolic rate (BMR) can inform us about the evolvability of the trait by providing estimates of heritability, and also of genetic correlations with other traits that may constrain the ability of BMR to respond to selection. Here, we studied a captive population of zebra finches (Taeniopygia guttata) in which selection lines for male courtship rate have been established. We measure BMR in these lines to see whether selection on male sexual activity would change BMR as a potentially correlated trait. We find that the genetic correlation between courtship rate and BMR is practically zero, indicating that the two traits can evolve independently of each other. Interestingly, we find that the heritability of BMR in our population $\left(h^{2}=0.45\right.$ ) is markedly higher than was previously reported for a captive zebra finch population from Norway. A comparison of the two studies shows that additive genetic variance in BMR has been largely depleted in the Norwegian population, especially the genetic variance in BMR that is independent of body mass. In our population, the slope of BMR increase with body mass differs not only between the sexes but also between the six selection lines, which we tentatively attribute to genetic drift and/or founder effects being strong in small populations. Our study therefore highlights two things. First, the evolvability of BMR may be less constrained by genetic correlations and lack of independent genetic variation than previously described. Second, genetic drift in small populations can rapidly lead to different evolvabilities across populations. Heredity (2013) 111, 175-181; doi:10.1038/hdy.2013.35; published online 1 May 2013
\end{abstract}

Keywords: heritability; metabolism; basal metabolic rate; quantitative genetics; genetic correlation; zebra finch

\section{INTRODUCTION}

Energy metabolism is a burgeoning area of research in evolutionary ecology, with major contemporary interest in understanding the causes and consequences of interindividual variation (Speakman et al., 2004; Burton et al., 2011; Konarzewski and Książek, 2013). One of the most commonly studied measures of energy metabolism is basal metabolic rate or BMR (White and Kearney, 2013), which is defined as the minimum energy requirement of an endothermic animal that is at rest, postabsorptive (that is, not digesting food) and thermoneutral during its normal period of inactivity (McNab, 1997). BMR is commonly viewed as the minimum energetic cost of selfmaintenance (Speakman et al., 2004), yet BMR is highly variable, often differing several fold even among individuals from the same population (Speakman et al., 2004; Burton et al., 2011). Such between-individual differences in the amount of energy required for basic self-maintenance may have important consequences for how much energy individuals have to allocate to other energetically demanding tasks, such as growth and reproduction (Burton et al., 2011). Consequently, the presence of large differences in metabolic rates among individuals from the same population is intriguing, and there is growing interest in understanding the adaptive significance of such variation (Speakman et al., 2004; Burton et al., 2011; Konarzewski and Książek, 2013).

In order to address questions regarding the adaptive significance of interindividual variation in BMR, it is necessary to know (a) whether variation in BMR is heritable and (b) the extent to which BMR is genetically correlated with other traits, which themselves might be under selection (Lande and Arnold, 1983; Tieleman et al., 2009; Careau et al., 2011). Quantitative genetic analyses of BMR can inform us about the evolvability of the trait by providing estimates of both narrow-sense heritability $\left(h^{2}\right)$ and of genetic correlations with other behavioural or morphological traits that may constrain the ability of BMR to respond to selection. Despite being one of the most widely measured physiological traits, there are relatively few studies to date that have explicitly investigated the heritability of BMR, and even fewer that have attempted to estimate genetic correlations between BMR and other traits (but see Nespolo et al., 2005; Rønning et al., 2007; Gebczynski and Konarzewski, 2009; Nilsson et al., 2009; Tieleman et al., 2009; Wone et al., 2009; Careau et al., 2011; Bushuev et al., 2012). Furthermore, these studies have produced conflicting results. For example, a genetic correlation of circa 1 was found between BMR and body mass in blue tits, Cyanistes caeruleus $(1.178 \pm 0.456$, mean \pm s.e.; Nilsson et al., 2009), while in piedflycatchers, Ficedula hypoleuca, the correlation did not differ from zero (0.087 \pm 0.297 , mean \pm s.e.; Bushuev et al., 2012). This has raised questions regarding the conditions under which findings can be generalised across studies (Bushuev et al., 2012).

Here, we study a captive population of zebra finches (Taeniopygia guttata) in which selection lines for male courtship rate have been established. The aims of this study were twofold. First, we replicated an earlier study on the genetics underlying BMR in zebra finches (Rønning et al., 2007), with the aim of shedding light on the extent to

${ }^{1}$ Evolutionary Ecology of Variation Group, Max Planck Institute for Ornithology, Seewiesen, Germany and ${ }^{2}$ Department of Behavioural Ecology and Evolutionary Genetics, Max Planck Institute for Ornithology, Seewiesen, Germany

Correspondence: Dr K Mathot, Evolutionary Ecology of Variation, Max Planck Institute for Ornithology, Eberhard Gwinner Strasse 7, Seewiesen, Bavaria 82319, Germany. E-mail: kmathot@orn.mpg.de

Received 19 September 2012; revised 5 March 2013; accepted 8 March 2013; published online 1 May 2013 
which the quantitative genetics of BMR differ in studies carried out on the same species, under similar conditions (laboratory). Second, we made use of the fact that our captive study population consists of six lines of birds that have been selected for divergent male courtship behaviour to evaluate whether selection on male sexual activity would change BMR as a potentially correlated trait. Correlational selection between BMR and behavioural traits have been hypothesised in several recent studies (Houston, 2010; Réale et al., 2010; Wolf and McNamara, 2012).

\section{MATERIALS AND METHODS}

\section{Breeding of selection lines}

The study was carried out on a captive population of domesticated zebra finches maintained at the Max Planck Institute for Ornithology (Seewiesen, Germany). This population has been studied over four consecutive generations (parental to F3) with regard to its sexual behaviour (Forstmeier et al., 2011). Male courtship rate, that is, the amount of male display towards a female in a standardised 5-min trial, had been measured in 583 males across these four generations (Forstmeier et al., 2011). On the basis of these data, breeding values for male courtship rate were calculated for all individuals in the population $(N=1219)$, including females, using pedigree-based animal models (see below)

From the pool of individuals that were still alive in May $2009(N=773)$, we established six different lines (two Control, two High and two Low lines). Each line was made up of 15 pairs that were set up in individual cages for breeding, but to be able to replace any bird that died during breeding, we kept another seven replacement birds of each sex. This means that on average 37.3 (rather than just 30) adults were set up for breeding for each line. Birds for the two Control lines (two times 22 birds of each sex) were selected randomly from the total pool of birds before the birds for the High and Low lines were chosen from the remainder based on their breeding values. For the High lines, we first selected the 30 birds of each sex with the highest breeding values, and randomly allocated half of them to each replicate line. After that, we picked the 14 replacement birds of each sex with the next highest breeding values and distributed them among the two lines. The two Low lines were set up in the same way, using the birds with the lowest breeding values for male courtship rate.

Pairs were allowed to breed until we obtained about 50 juveniles from each line (cohort 1), after which we swapped the breeding partners within each line and allowed the newly formed pairs to raise another 50 juveniles per line (cohort 2). The allocation of pair members was carried out in such a way as to standardise the average inbreeding coefficient across the six lines. In the most inbred line (High 2), we attempted to minimise inbreeding as much as possible, and pairings in the five other lines were chosen to match the mean value for this line. The mean inbreeding coefficient of the first generation of offspring (referred to as S1) was $\mathrm{F}=0.021$, and $\mathrm{F}=0.067$ for the second generation (S2), based on a six- and seven-generation pedigree, respectively.

In each generation we produced about 600 offspring (two cohorts times 50 offspring per line). These were reared in large mixed-sex peer groups (of about 150 birds) kept in aviaries from day 35 of age (independent from parents) to about day 113 (sexual maturity; s.d.: 6.3 days; range: 95-130). After that, birds were maintained in unisex groups, either in flocks of about 150 or during periods of measurement (of courtship rate and BMR), in groups of two in cages. After assessing courtship rate of all male offspring (each having two trials at $118 \pm 6$ days s.d. of age and another two trials at $212 \pm 6$ days s.d. of age), we calculated new breeding values based on all data available. The next generation of breeders was selected from the pool of about 100 offspring per line either randomly (Control lines) or based on breeding values (High and Low lines).

For the study of BMR, we waited until the S1 generation had finished breeding the first and second cohorts of the S2 generation. The S1 breeding pairs were then split up and kept for $86 \pm 36$ days s.d. (range 12-128 days) in unisex groups of two in cages until the measurement of BMR. Of the S2 generation, we measured BMR in the entire first cohort ( $n=311$ offspring) shortly after the second set of courtship rate measurements (at $246 \pm 24$ days s.d. of age), and of the $\mathrm{S} 1$ generation, we measured BMR of all living parents of these birds ( $n=135$ parents; at $773 \pm 119$ days s.d. of age).

\section{BMR measurements}

Metabolic rate measurements of zebra finches were carried out between October 2011 and April 2012 on a total of 446 individuals. For at least 12 days before metabolic rate measurements, zebra finches were housed in same sex pairs in cages $\left(60 \times 40 \times 45 \mathrm{~cm}^{3}\right.$ high $)$ under a constant $14 \mathrm{~h}$ light: $10 \mathrm{~h}$ dark photoperiod. Zebra finches were removed from their cages circa $1 \mathrm{~h}$ before lights out (between 1815 and 1915 hours) and weighed to the nearest $0.1 \mathrm{~g}$ before being placed in a metabolic chamber made from metal cylinders with airtight lids. Metabolic rates were measured overnight for up to 9 individuals per night, and the following morning (between 0500 and 0600 hours) birds were removed from the metabolic chambers and returned to their home cages. We did not use activity detectors, although periods of activity could be inferred from peaks in $\mathrm{O}_{2}$ consumption. Birds were measured in random order with respect to their courtship rate selection line status.

BMR was measured as $\mathrm{O}_{2}$-consumption rates using three identical setups. Each set up consisted of a four-channel open flow respirometry system with water vapour, $\mathrm{CO}_{2}$ and $\mathrm{O}_{2}$ analysers. $\mathrm{H}_{2} \mathrm{O}$ and $\mathrm{CO}_{2}$ were removed from influent air using Drierite and Ascarite, and the air was then pumped through three metabolic chambers made from 1-1 metal cylinders with airtight lids. The chambers were kept in an environmental chamber (Binder KB53 Refrigerated Incubator, Binder $\mathrm{GmbH}$, Tuttlingen, Germany), which maintained the chambers at a constant temperature of $35.0 \pm 0.1{ }^{\circ} \mathrm{C}$, which is within the thermoneutral zone of the zebra finch (Calder, 1964). A constant air-flow rate into the chambers of $200 \mathrm{ml} \mathrm{min}^{-1}$ was maintained using mass-flow controllers (Sable Systems, Las Vegas, NV, USA). The $\mathrm{O}_{2}, \mathrm{H}_{2} \mathrm{O}$ and $\mathrm{CO}_{2}$ concentrations in effluent air streams were measured using a water vapour analyser (Sable Systems) and oxygen and $\mathrm{CO}_{2}$ analysers (FoxBox; Sable Systems). An additional stream of dry, $\mathrm{CO}_{2}$ free air was used as a baseline throughout recordings. An automatic valve switched between streams, so that $10 \mathrm{~min}$ of baseline $\mathrm{O}_{2}$ concentrations were recorded between every 30-min recording of a zebra finch. Thus, on each of the three identical respirometry set-ups, $130 \mathrm{~min}$ of recording were made for each complete cycle (four 10-min baseline recordings and three 30-min zebra finch recordings), and an average of five complete cycles were recorded for each set-up per night (that is, five 30min recordings per bird).

Analogue outputs from the $\mathrm{H}_{2} \mathrm{O}, \mathrm{O}_{2}$ and $\mathrm{CO}_{2}$ analysers were fed to a computer via a 16-bit A/D converter card. $\mathrm{H}_{2} \mathrm{O}, \mathrm{O}_{2}$ and $\mathrm{CO}_{2}$ concentrations were recorded at 1-s intervals. The rate of $\mathrm{O}_{2}$ consumption $\left(\mathrm{VO}_{2}\right)$ was calculated following Lighton (2008). Briefly, $\mathrm{VO}_{2}$ was calculated as:

$$
V \mathrm{O}_{2}=F R \times\left(\left(F_{i} \mathrm{O}_{2}-F_{\mathrm{e}} \mathrm{O}_{2}^{\prime}\right)-F_{\mathrm{e}} \mathrm{O}_{2}^{\prime} *\left(F_{\mathrm{e}} \mathrm{CO}_{2}^{\prime}\right)\right) /\left(1-F_{\mathrm{e}} \mathrm{O}_{2}^{\prime}\right)
$$

where $\mathrm{FR}$ is the flow rate of dry, $\mathrm{CO}_{2}$ free air into the metabolic chambers (in $\left.\mathrm{ml} \mathrm{min}{ }^{-1}\right), F_{\mathrm{i}} \mathrm{O}_{2}$ is the partial pressure of $\mathrm{O}_{2}$ in the influent air, $F_{e} \mathrm{O}_{2}^{\prime}$ is the partial pressure of $\mathrm{O}_{2}$ in the effluent air after correcting for dilution due to water vapour pressure and $\mathrm{F}_{e} \mathrm{CO}_{2}^{\prime}$ is the partial pressure of $\mathrm{CO}_{2}$ in the effluent air after correcting for water vapour pressure dilution. Water vapour pressure dilution corrections were performed as follows:

$$
\begin{aligned}
& F_{\mathrm{e}} \mathrm{O}_{2}^{\prime}=F_{\mathrm{e}} \mathrm{O}_{2} \times \mathrm{BP} /(\mathrm{BP}-\mathrm{WVP}) \\
& F_{\mathrm{e}} \mathrm{CO}_{2}^{\prime}=F_{\mathrm{e}} \mathrm{CO}_{2} \times \mathrm{BP} /(\mathrm{BP}-\mathrm{WVP})
\end{aligned}
$$

where $F_{\mathrm{e}} \mathrm{O}_{2}$ and $\mathrm{F}_{\mathrm{e}} \mathrm{CO}_{2}$ are the uncorrected values of $\mathrm{O}_{2}$ and $\mathrm{CO}_{2}$ concentrations measured in the effluent air, $\mathrm{BP}$ is the barometric pressure $(\mathrm{kPa})$ and WVP is the water vapour pressure $(\mathrm{kPa})$. We used ExpeDataPro (Sable Systems) to select and calculate the lowest $10 \mathrm{~min}$ average $\mathrm{VO}_{2}$, and this was used to represent the BMR.

\section{Measurements of morphology and courtship rate}

For the quantitative genetic analyses, we made use of most of the morphological data available for our study population (2515 birds: 227 parental, 304 F1, 518 F2, 147 F3, 129 F4, 564 S1 and 626 S2 generation). Measurements of body mass (to the nearest $0.1 \mathrm{~g}$ ), tarsus length (to the nearest $0.1 \mathrm{~mm}$ ) and wing length (to the nearest $0.5 \mathrm{~mm}$ ) were nearly always taken when birds reached sexual maturity (around days 100-120), but repeated measures from other occasions were also available (see Supplementary Table S1 for sample sizes). To account for various confounding factors, we kept track of the birds' 
age at measurement (mean: 232 days; s.d.: 269 days; range: 32-1177 days), time of day (range: 08:22-20:26 hours), observer (nine levels) and measurement session (measurements taken at 30 different occasions, with between 72 and 621 birds measured per occasion).

Measurements of male courtship rate were carried out for a total of 1254 males (in 5582 test trials), following the previously described protocol (Forstmeier, 2004). We recorded variation in male age (mean: 220 days; s.d.: 124 days; range: 93-646 days), time of day (range: 08:27-18:19 hours) and measurement session (17 sessions with 148 to 832 trials per session). All measurements were taken by one of two different observers (WF for parental to F4, and KM for S1 and S2). Repeated measurements on the same male within a session were always carried out on 2-8 consecutive days, and measurement day was accounted for statistical significance.

The morphological measures were approximately normally distributed. Courtship rate was square-root transformed to approach normality, yet the distribution was somewhat zero-inflated (20.7\% of measurements were zero).

\section{Estimation of genetic parameters}

To partition the phenotypic variance into its causal components, we used a restricted maximum likelihood model run in VCE 6.0.2 (Groeneveld et al., 2008). All models are based on an error-free pedigree (verified by large numbers of markers) comprising 2723 birds (including the grand-parental generation that is without phenotypes). In the first generations (parental to F2) nearly all of the birds had been cross-fostered at the egg stage among broods, but afterwards (F3 to S2) cross-fostering had been abandoned, as the rearing environment did not seem to have noteworthy effects on sexual behaviour or morphology (Forstmeier et al., 2011). However, we cannot be sure that this absence of effect also applies to BMR, which was only measured in non-crossfostered birds. Hence, it is possible that the estimated heritability is inflated by early environmental effects.

For all traits with multiple measures (courtship rate and morphology), phenotypic variances were decomposed into additive genetic variance, permanent environment variance and residual variance. For BMR, where we had only one measure per individual, we separated the additive genetic variance from the residual variance. For this study, five different models were fitted:

(1) A single-trait permanent environment model for courtship rate, for the estimation of breeding values that were used for breeding the selection lines. This model was updated with the new phenotypic data after each new generation.

(2) A sex-specific four-trait permanent environment model for male BMR, female BMR, male body mass and female body mass. This was performed to check for between-sex genetic correlations and for sex differences in the relationships between BMR and body mass. This model, however, had a low power for detecting sex-specific effects because BMR data were available for only 220 females and 226 males. We therefore present the output only in the Supplementary Information (Supplementary Table S3).

(3) A five-trait permanent environment model for courtship rate, wholeorganism BMR, body mass, wing length and tarsus length. In this and the previous model, the body mass measurements obtained on the evening before BMR measurement were included in the total body mass data, and by entering them in the same rows as the BMR data we also obtain an estimate for the residual covariance between BMR and body mass. Wholeorganism BMR was used to allow direct comparisons with the results of the Rønning et al. (2007) study. The results of this model are presented below.

The following two models are modified versions of that main model, and are only presented in the Supplementary Information.

(4) Like model 3, but including mother identity (not linked to the pedigree) as an additional variance component. The resulting maternal effect estimates, and especially the maternal effect covariances (Supplementary Table S5) should be regarded with caution, because the breeding design is not optimal for quantifying maternal effects (only 24\% of the birds with BMR data has either a maternal or a paternal half-sib that was also measured for BMR).
(5) Like model 3, but replacing BMR and body mass by a single-trait, residual BMR, from sex-specific ordinary least-square (OLS) regressions over body mass. This model was included to estimate the heritability of residual BMR (Supplementary Table S6), which is a frequently used trait besides whole-organism BMR that we consider in models 2-4.

In all five models we used the following parameters as fixed effects: inbreeding coefficient F (calculated by Pedigree Viewer 6.4a; Kinghorn and Kinghorn, 2010), age, measurement session, sex (for all traits except courtship rate), time of day (for mass and courtship rate), observer (for tarsus and wing) and test day (for courtship rate). For model 3, all obtained parameter estimates for fixed effects were compared with estimates from simple mixed-effect models (lme4 package) run in R 2.15.1 (see Supplementary Table S4). Measuring session was fitted as a fixed (rather than random) effect, as this helped with model convergence and we were not interested in betweentrait covariances of session effects. All models converged with VCE status 1 (optimal). Parameter estimates that are more than 2 s.e. away from zero are considered significant (in the sense of a label of confidence in the estimate).

From the obtained variance-covariance matrices, we calculated heritabilities, conditional heritabilities, as well as coefficients of additive genetic and residual variance, all in the same way as described in detail by Rønning et al. (2007). The estimation of quantitative genetic parameters and the estimation of genetic correlations in particular require large sample sizes. We therefore refrain from attempting line-specific analyses.

\section{OLS versus RMA regression}

To examine the phenotypic variance in BMR in relation to body mass and as a function of sex and selection line, we used general linear models run in SPSS 18.0. Such models based on OLS regression minimise the residual variance in the dependent trait (BMR) while ignoring that the predictor variable (here: body mass) also contains biological noise that could even be partly influenced by BMR. For instance, birds with intrinsically high BMR might put on more weight in order to survive overnight. Hence, one should be aware that in such cases OLS regression introduces some predictable biases, such as an overestimation of sexual dimorphism in favour of the larger sex (Forstmeier, 2011). Reduced major axis (RMA) regression is probably the most widely used alternative line fitting method, but it assumes equal amounts of biological noise in both variables, that is, a symmetrical relationship between body mass and BMR. We consider this scenario unlikely, as BMR seems to depend on mass in a much more direct way than mass would depend on BMR. In line with this, it seems that the practically equivalent method of calculating a ratio (that is, expressing BMR as oxygen consumption per gram of body mass) has been abandoned in the more recent literature. Nevertheless, we used the 'smatr' package in R (Warton and Ormerod, 2007) to fit RMA regression lines and to test their slopes and intercepts for sex differences. The purpose of this was to see the direction in which parameter estimates change as one gradually moves from OLS regression lines towards RMA regression lines. In these models, the covariate body mass was centred following Schielzeth (2010) to facilitate the interpretation of main effects in the presence of interaction terms.

\section{RESULTS}

Phenotypic analyses of BMR in relation to sex and line

A summary of means, s.d. and sample sizes for all measured phenotypic traits in relation to sex and selection line can be found in Supplementary Table S1. BMR was significantly higher in females than in males ( $t$-test with unequal variances: $b=0.0341, t_{418.4}=3.38$, $P=0.0008$ ), and also significantly more variable in females than in males (Levene's test for equality of variances: $\mathrm{F}=8.6, P=0.0035$ ). The six selection lines did not differ significantly in their average BMR (ANOVA: $F_{5,440}=1.47, P=0.20$ ). However, these patterns change when considering BMR as a function of body mass (measured in the evening immediately before BMR measurement), as the sexes and the lines differed significantly in their body mass (Supplementary Table S1). 
A full model of BMR as a function of sex and line in relation to mass (allowing for different slopes of BMR over mass for the sexes and lines) is shown in Table 1. As indicated by the significant interaction terms, BMR increased more steeply with mass in females than in males (Figure 1; $P=0.016$ ), and increased more steeply in the Control line 1 than in any of the other lines (Figure 2; d.f. $=5$, $P=0.006$ ). In this model, the main effect of sex (estimated where the centred covariate mass is zero, that is, for the population average of mass) is reduced to $b=0.0156(P=0.047)$. However, OLS regression tends to overestimate sexual dimorphism in favour of the larger sex (Forstmeier, 2011), here females. Accordingly, the sexual dimorphism

Table 1 Between-individual differences in $\mathrm{BMR}\left(\mathrm{ml} \mathrm{O}_{2}\right.$ consumption per min) as a function of body mass (scaled to a mean of zero and unity s.d.), sex and selection line

\begin{tabular}{|c|c|c|c|c|c|}
\hline Source & $\begin{array}{c}\text { Type III sum of } \\
\text { squares }\end{array}$ & d.f. & $\begin{array}{l}\text { Mean } \\
\text { square }\end{array}$ & $F$ & P-value \\
\hline Corrected model & 2.354 & 14 & 0.168 & 26.080 & $<0.0001$ \\
\hline Intercept & 231.129 & 1 & 231.129 & 35848.452 & $<0.0001$ \\
\hline Session & 0.317 & 1 & 0.317 & 49.126 & $<0.0001$ \\
\hline Sex & 0.026 & 1 & 0.026 & 3.975 & 0.047 \\
\hline Line & 0.066 & 5 & 0.013 & 2.036 & 0.073 \\
\hline Centred mass & 1.324 & 1 & 1.324 & 205.311 & $<0.0001$ \\
\hline $\begin{array}{l}\text { Sex } \times \text { centred } \\
\text { mass }\end{array}$ & 0.038 & 1 & 0.038 & 5.897 & 0.016 \\
\hline $\begin{array}{l}\text { Line } \times \text { centred } \\
\text { mass }\end{array}$ & 0.106 & 5 & 0.021 & 3.293 & 0.006 \\
\hline Error & 2.779 & 431 & 0.006 & & \\
\hline Total & 322.229 & 446 & & & \\
\hline Corrected total & 5.133 & 445 & & & \\
\hline
\end{tabular}

Abbreviations: BMR, basal metabolic rate; d.f., degrees of freedom.

The factor Session accounts for the deviating BMR measurements from Session C (see Supplementary Figure S1 and Supplementary Table S2).

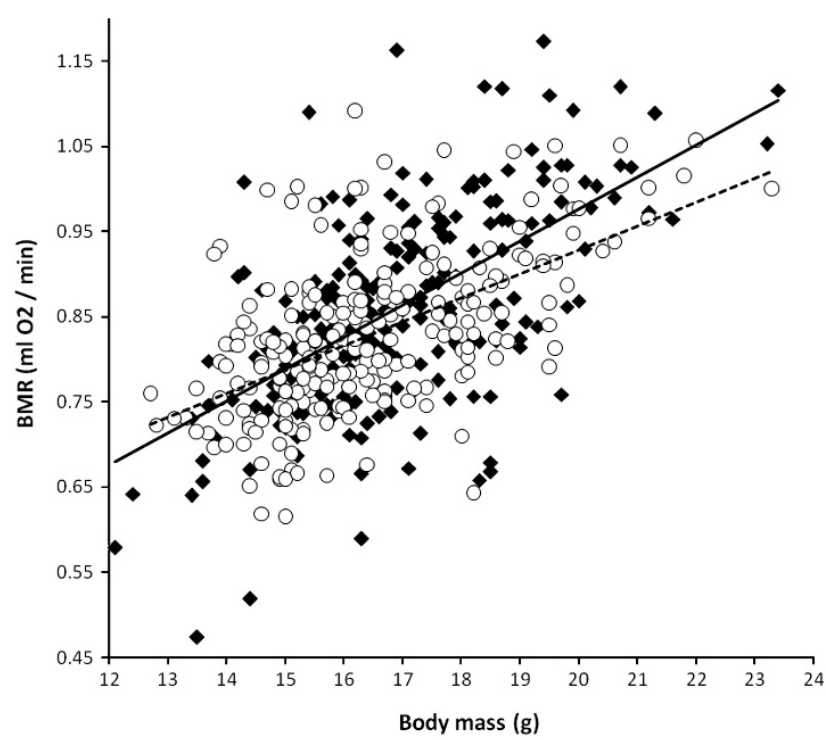

Figure $1 \mathrm{BMR}$ in relation to body mass measured at dusk for female (solid diamonds) and male (open circles) zebra finches. OLS regression lines are shown for females (full line: $y=0.03755 x+0.2257 ; r=0.606$ ) and males (hatched line: $y=0.02800 x+0.3676 ; r=0.562$ ). in BMR is further reduced (to $b=0.0050$ ) and clearly nonsignificant $(P=0.59)$ when extracting it from fitted RMA regression lines (shown in Supplementary Figure S2). However, the difference in slopes between the sexes is still significant $(P=0.005)$ when using RMA regression (Supplementary Figure S2). The between-line differences in BMR related to mass (estimated at the population mean of mass) are still short of significance in the OLS-based model $\left(\mathrm{F}_{5,431}=2.04, P=0.073\right.$; Table 1$)$.

Finally, we tested whether BMR is higher for birds that grew up in larger broods (as found by Verhulst et al., 2006). Natal brood size (non-manipulated number of chicks in a brood surviving to day 8 ) varied substantially (brood size $\times N$ : $1 \times 35,2 \times 104,3 \times 118,4 \times 125$, $5 \times 45,6 \times 19$ ), but did not affect BMR when added as a covariate to the full model shown in Table $1\left(b=-0.002, \mathrm{~F}_{1,430}=0.3, P=0.57\right)$.

Quantitative genetic analyses of BMR in relation to courtship rate and body size

A four-trait sex-specific model (male mass, female mass, male BMR and female BMR) indicated a high genetic correlation between male and female BMR $\left(r_{\mathrm{A}}=0.91 \pm 0.16\right)$ and no marked sex differences in the genetic correlations between BMR and mass (Supplementary Table S3). In the following, we therefore focus on parameter estimation for the population as a whole, with sex accounted for only as a fixed effect.

BMR was independent of age and inbreeding coefficient (parameter estimates for fixed effects are presented in Supplementary Table S4). Table 2 shows the additive genetic variance components, the permanent environment components (that is, the individually repeatable, but not additive genetic components), as well as the residual variance components for all five traits, together with all between-trait correlations.

The heritability (of a single measurement) of BMR was rather high ( $h^{2}=0.45 \pm 0.06$ ) compared with the $h^{2}=0.25$ found by Rønning et al. (2007).

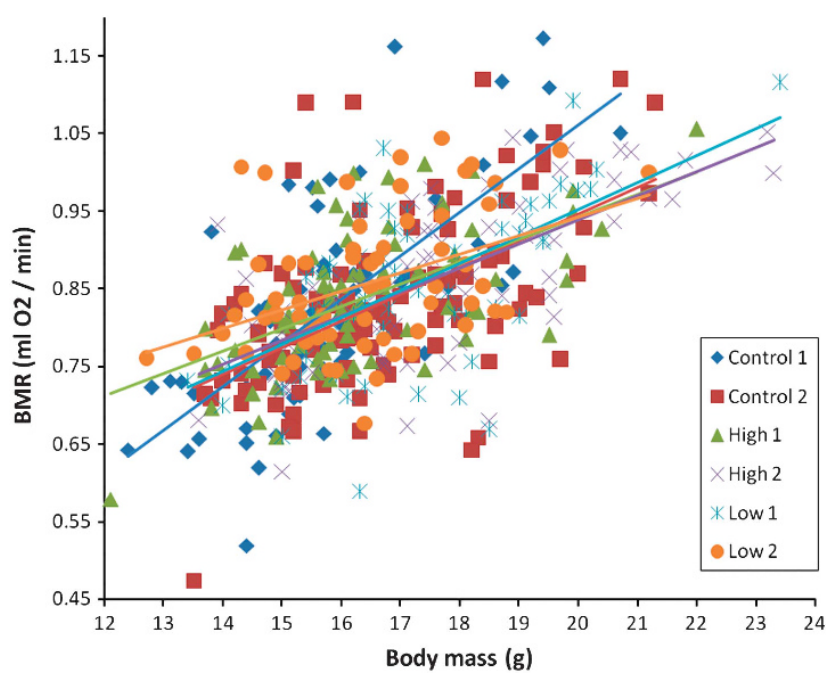

Figure 2 BMR in relation to body mass measured at dusk shown separately for each selection line. OLS regression lines are shown for Control line 1 (dark blue: $y=0.0563 x-0.0637 ; r=0.711$ ), Control line 2 (red: $y=0.0344 x+0.2581 ; r=0.558$ ), High line 1 (green: $y=0.0288 x+$ $0.3661 ; r=0.573$ ), High line 2 (purple: $y=0.0310 x+0.3181 ; r=0.666$ ), Low line 1 (light blue: $y=0.0349 x+0.2554 ; r=0.612$ ), Low line 2 (orange: $y=0.0244 x+0.4567 ; r=0.434$ ). 
Table 2 Variance components and correlations estimated from a five-trait permanent environment animal model performed in VCE

\begin{tabular}{|c|c|c|c|c|c|}
\hline & Courtship rate & $B M R$ & Mass & Tarsus & Wing \\
\hline \multicolumn{6}{|l|}{ Genetic } \\
\hline Courtship rate & $0.244 \pm 0.022$ & $-0.036 \pm 0.054$ & $-0.013 \pm 0.040$ & $0.004 \pm 0.057$ & $-0.048 \pm 0.049$ \\
\hline Mass & & & $0.463 \pm 0.026$ & $0.385 \pm 0.043$ & $0.277 \pm 0.041$ \\
\hline Tarsus & & & & $0.641 \pm 0.020$ & $0.305 \pm 0.032$ \\
\hline Wing & & & & & $0.699 \pm 0.014$ \\
\hline Courtship rate & $0.318 \pm 0.036$ & - & $0.161 \pm 0.036$ & $0.109 \pm 0.053$ & $0.104 \pm 0.079$ \\
\hline BMR & & - & - & - & - \\
\hline Mass & & & $0.194 \pm 0.016$ & $0.653 \pm 0.055$ & $0.603 \pm 0.084$ \\
\hline Tarsus & & & & $0.246 \pm 0.019$ & $0.771 \pm 0.053$ \\
\hline Wing & & & & & $0.097 \pm 0.019$ \\
\hline \multicolumn{6}{|l|}{ Residual } \\
\hline Tarsus & & & & $0.113 \pm 0.007$ & $0.079 \pm 0.037$ \\
\hline Wing & & & & & $0.204 \pm 0.010$ \\
\hline
\end{tabular}

Abbreviation: BMR, basal metabolic rate.

Variance components are standardised by the phenotypic variance (after controlling for fixed effects). Variance components \pm s.e.m. are shown on the diagonal (heritabilities are in bold),

correlations \pm s.e.m. between pairs of traits are shown off the diagonal. The additive genetic and permanent environment components together reflect the individual repeatability of single units of

observation. Parameters that cannot be estimated from our data are marked with "-.'

Table 3 Estimates of phenotypic mean, phenotypic s.d., additive genetic variance $\left(V_{A}\right)$, residual variance $\left(V_{R}\right.$; here including permanent environmental variance), heritability $\left(h^{2}\right)$ and its s.e., coefficient of additive genetic variance $\left(C_{A}\right)$ and of residual variance $\left(C_{R}\right)$ from our study, and for comparison, the estimates from the Rønning et al. (2007) study on a captive zebra finch population from Norway

\begin{tabular}{|c|c|c|c|c|c|c|c|c|c|}
\hline Trait & Phen. mean (s.d.) & $\mathrm{V}_{A}$ & $\mathrm{~V}_{R}$ & $\mathrm{~h}^{2}$ (s.e.) & $C V_{A}$ & $C V_{R}$ & $\mathrm{~h}^{2}$ (s.e.) Rønning & $C V_{A}$ Rønning & $C V_{R}$ Rønning \\
\hline Courtship rate & $3.70(2.50)$ & 1.184 & 3.676 & $0.244(0.022)$ & 29.41 & 51.82 & & & \\
\hline BMR & $0.843(0.107)$ & 0.00453 & 0.00555 & $0.449(0.063)$ & 7.98 & 8.84 & $0.250(0.043)$ & 4.91 & 8.50 \\
\hline Mass & $16.58(2.05)$ & 1.435 & 1.664 & $0.463(0.026)$ & 7.23 & 7.78 & $0.336(0.059)$ & 5.95 & 8.36 \\
\hline Tarsus & $17.12(0.58)$ & 0.221 & 0.124 & $0.641(0.020)$ & 2.74 & 2.05 & $0.322(0.046)$ & 2.45 & 3.55 \\
\hline Wing & $58.29(1.58)$ & 1.506 & 0.649 & $0.699(0.014)$ & 2.11 & 1.38 & $0.477(0.046)$ & 2.04 & 2.14 \\
\hline
\end{tabular}

Abbreviation: BMR, basal metabolic rate.

Courtship rate is measured in square-root transformed seconds, BMR in $\mathrm{ml} \mathrm{O}_{2}$ consumption per min, mass in $\mathrm{g}$ and tarsus and wing length in $\mathrm{mm}$.

The genetic correlation between BMR and courtship rate was estimated to be practically zero $\left(r_{\mathrm{A}}=-0.036 \pm 0.054\right)$, indicating that selection on courtship rate should not lead to a correlated response in BMR. In contrast, the genetic correlation between BMR and body mass was estimated to be very high $\left(r_{\mathrm{A}}=0.87 \pm 0.04\right)$, indicating that the two traits constrain each other's evolvability. The conditional heritability of BMR on body mass was estimated as 0.11 , which corresponds to $25 \%$ of its unconditional heritability. This estimate of conditional heritability is almost three times higher than the estimate of 0.041 found by Rønning et al. (2007). Similar G-matrix estimates were obtained from models that accounted for maternal effects (Supplementary Table S5). A model using residual BMR as a trait (residuals from Figure 1) yielded a heritability estimate of $h^{2}=0.18 \pm 0.06$ (Supplementary Table S6).

For all traits, heritability estimates from our study population are markedly higher than those obtained from the Norwegian zebra finch population, but the causes for the differences in variances are not the same for all traits (Table 3). For BMR and body mass, the lower heritabilities in the Norwegian population primarily result from reduced additive genetic variance for these traits in Norway. In contrast, for tarsus and wing length, the lower heritability primarily stems from increased residual variance (for example, due to more measurement error) in the Rønning et al. (2007) study.

\section{DISCUSSION}

Here, we provide quantitative genetic estimates of BMR in a captive population of zebra finches. This study closely mirrors previous work in another population of captive zebra finches (Rønning et al., 2007) with the aim of shedding light on possible sources of differences between previous quantitative genetics studies of BMR. Consistent with the findings of Rønning et al. (2007), we observed significant heritabilities for BMR, body mass, tarsus and wing length. However, 
in all cases, the heritabilities observed in our study were higher (Table 3). We also observed significant genetic correlations between BMR and these morphological traits, although in contrast with the previous study in zebra finches, we found greater amounts of additive genetic variation in BMR that is independent of body mass (conditional $h^{2}=0.11$ ), indicating that BMR could also evolve independently of body mass in our population.

In addition, we investigated the genetic relationship between BMR and courtship behaviour, following earlier suggestions that BMR may coevolve with various behavioural traits (Houston, 2010; Wolf and McNamara, 2012), including behavioural traits related to reproduction (Réale et al., 2010). Although courtship rate showed a moderate heritability $\left(h^{2}=0.24\right)$, the genetic correlation between courtship rate and BMR was estimated to be practically zero, indicating that BMR and courtship activity can evolve independently of one another.

The heritability of BMR found in our study $\left(h^{2}=0.45\right)$ was higher than that reported in an earlier study in the same species $\left(h^{2}=0.25\right.$; Rønning et al., 2007) and closer to the heritabilities previously reported for free-living passerines (blue tits, Cyanistes caeruleus, $h^{2}=0.59$ (Nilsson et al., 2009), and pied-flycatchers, Ficedula hypoleuca, $h^{2}=0.43$, (Bushuev et al., 2012)). This suggests that between-study differences in the quantitative genetics of BMR are not merely the result of a dichotomy between lab versus field populations, as has previously been suggested (Bushuev et al., 2012). The higher heritability of BMR observed in our study compared with the previous study in zebra finches was largely due to a higher additive genetic variance in our population. We also observed higher heritabilities of morphological traits in our study compared with Rønning et al. (2007) (Table 3). While the higher heritability estimate for body mass in our population also came about via higher additive genetic variance for this trait in our population, the higher heritability for tarsus and wing length in our population came about primarily from a lower residual variance. Thus, differences in the heritability of traits between our population and the Norwegian population of zebra finches cannot be universally attributed to a depletion of genetic variance in the Norwegian zebra finch population.

There was a significant genetic correlation between body mass and BMR $(0.87 \pm 0.04)$ in our zebra finch population. Nonetheless, there is moderate scope for BMR to evolve independently of body mass as $\mathrm{BMR}$ conditioned on the genetic variance in body mass was estimated as $h^{2}=0.11$. These estimates differ from those of a Norwegian population of zebra finches where there appears to be a more limited scope for BMR to evolve independently of body mass (conditional $h^{2}=0.04$, genetic correlation with body mass $\left.=0.91 \pm 0.08\right)$ (Rønning et al., 2007).

We are aware of only two other studies in birds that have estimated the genetic correlation between body mass and BMR. A study in freeliving pied-flycatchers found no evidence of a genetic correlation between BMR and body mass (Bushuev et al., 2012). Another study on captive stonechats (Saxicola torquata ssp.) found a significant genetic correlation between BMR and body mass in birds from a Kenyan population ( $S$. torquata axillaris: $r_{\mathrm{A}} \pm$ s.e $=0.78 \pm 0.36$ ), but not in birds from an Austrian population ( $S$. torquata rubicola: $0.40 \pm 0.35$ ) (Tieleman et al., 2009). However, as the authors themselves point out, the comparisons between populations of stonechats were based on very small sample sizes for these types of analyses $(N=14$ and 45 , respectively), and should therefore be interpreted with caution. However, taken together, the results of these three studies and this study suggest that patterns of genetic covariance between traits may be population specific, making it difficult to infer general patterns from a limited number of studies, including studies on the same species.

Although the reasons for strong population-specific differences between our study and that of Rønning et al. (2007) are unknown, we suggest that such patterns may emerge, in part, because of strong genetic drift and/or founder effects in small populations (Roff, 2000; Jones et al., 2003). Support for this interpretation comes from the finding that the phenotypic correlation between BMR and body mass differed significantly among our selection lines (Control line 1 versus other lines). This difference probably arose simply from randomly selecting a different set of individuals as founders of this control line. We are aware of at least one study in house mice (Mus domesticus) in which significant phenotypic divergence in metabolic traits were observed between replicate selection lines for locomotory activity (Kane et al., 2008). Although our study and that of Kane et al. (2008) both provide evidence for a potentially important role of genetic drift in laboratory populations, genetic drift and founder effects may also have important effects in small or isolated wild populations. In fact, although numerous studies in free-living populations have attributed population-specific differences in the quantitative genetics estimates of traits to selection, the majority of these have not ruled out the role of drift in generating the degree of divergence observed (reviewed in Garland Jr and Carter, 1994). Although drift should generate proportional changes in $G$-matrices on average, it may lead to substantial differences in estimates between any given pair on populations (Phillips et al., 2001). The large differences in estimates between our study and that of Rønning et al. (2007) highlight the need for more replicated studies on the quantitative genetics of BMR before general patterns of the underlying genetic architecture can be inferred.

We also investigated whether there was any evidence of genetic correlations between BMR and courtship behaviour in our population, following the suggestion of several recent theoretical studies that adaptive individual differences in BMR may come about via coevolution of physiological and behavioural traits when particular combinations of behaviours and physiological traits can achieve equal fitness (Houston, 2010; Wolf and McNamara, 2012). For example, individuals with higher BMR may achieve equal fitness to individuals with low BMR if the additional metabolic costs they pay are offset by increased expression of other fitness-enhancing traits, such as courtship behaviour (to increase reproductive success) or exploration or activity behaviours (to increase rates of food discovery) (Biro and Stamps, 2010; Réale et al., 2010). Although we estimated significant heritabilities for both BMR and courtship rate, we found no evidence for a genetic correlation between these two traits, indicating that they are free to evolve independently in our population of zebra finches. It could be argued that in laboratory reared animals with ad libitum access to food, the costs of having a higher metabolic rate may be negligible, resulting in an uncoupling of BMR and other fitnessrelated traits over several generations of breeding. However, this does not appear to be universally true, as we are aware of at least two studies that have found significant genetic correlations between resting metabolic rate and behaviour in captive populations of mammals (spontaneous activity in Swiss-Webster laboratory mice (Gebczynski and Konarzewski, 2009), and exploration behaviour in a Peromyscus population recently derived from wild stock (Careau et al., 2011)).

Despite high statistical power to detect phenotypic correlations between metabolic rate and other traits (due to the large sample size of our study, $N=446$ individuals), we were unable to replicate results from two earlier studies in zebra finches. In one study, individuals 
raised in experimentally enlarged broods (5-6 young) had significantly higher metabolic rates than those raised in reduced broods (2-3 young) (Verhulst et al., 2006). Although the range of natural brood sizes in our experiment was comparable to those in the previous study (range: 1-6), we found no effect of brood size on BMR $\left(b=-0.002, \mathrm{~F}_{1,430}=0.3, P=0.57\right)$. We cannot discount the possibility that our results differ from those of Verhulst et al. (2006) because of different effects of natural brood size versus experimentally manipulated brood size on metabolic rates. However, at least one other study in passerines (blue tits) found no effect of experimentally manipulated rearing environment (reduced versus enlarged brood size) on metabolic rate (Nilsson et al., 2009), providing further support for our assertion that general patterns cannot be inferred from unreplicated studies. In another study, inbred female zebra finches (produced from full-sib matings, $\mathrm{F} \approx 0.25$ ) had significantly higher metabolic rates than outbred females (produced from matings between unrelated individuals, $\mathrm{F} \approx 0.0$ ). In contrast, we found no evidence for any effect of inbreeding ( $F$ range: $0-0.25$, including 90 individuals with $\mathrm{F} \geqslant 0.125)$ on $\mathrm{BMR}$ in our study population (Supplementary Table S4).

\section{CONCLUSIONS}

Earlier studies on the quantitative genetics of BMR have yielded markedly different results, but it was unclear to what extent these differences reflected species-specific patterns, general differences between captive versus free-living animals or population-specific differences in the quantitative genetics of BMR (Tieleman et al., 2009; Bushuev et al., 2012). We replicated an earlier study in zebra finches and found that the quantitative genetics estimates of BMR and morphological traits on the same species under similar conditions differed markedly between populations. Our results reveal that discrepancies between earlier studies cannot solely be accounted for by species-specific differences or to differences between captive versus free-living animals. Our study suggests that these traits and the phenotypic and genetic correlations among them may change relatively rapidly between populations. This highlights the need for more replicated studies of the quantitative genetics of metabolism to allow for general patterns to be elucidated.

\section{DATA ARCHIVING}

Data have been deposited at Dryad: doi:10.5061/dryad.rt61j.

\section{CONFLICT OF INTEREST}

The authors declare no conflict of interest.

\section{ACKNOWLEDGEMENTS}

We thank Anjuli Barber and Judit Mateos for help with catching and handling zebra finches, Sonja Bauer, Edith Bodendorfer, Annemarie Grötsch, Monika Halser, Petra Neubauer, Andrea Peter, Frances Weigel and Barbara Wörle for animal care, and two anonymous referees for comments on an earlier version of the manuscript. KJM was supported by an Alexander von Humboldt postdoctoral fellowship, and BK, KM and WF were supported by the Max Planck Society. This experiment complied with local laws and was carried out under the housing and breeding permit no. 311.4-si (by Landratsamt Starnberg, Starnberg, Germany).
Biro PA, Stamps JA (2010). Do consistent individual differences in metabolic rate promote consistent individual differences in behavior? Trends Ecol Evol 25: 653-659.

Burton T, Killen SS, Armstrong JD, Metcalfe NB (2011). What causes intraspecific variation in resting metabolic rate and what are its ecological consequences? Proc $R$ Soc Lond Ser B 278: 3465-3473.

Bushuev AV, Husby A, Sternberg H, Grinkov VG (2012). Quantitative genetics of basal metabolic rate and body mass in free-living pied flycatchers. J Zool 288: 245-251.

Calder WA (1964). Gaseous metabolism and water relations of the zebra finch, Taeniopygia castanotis. Physiol Zool 37: 400-413.

Careau V, Thomas D, Pelletier F, Turki L, Landry F, Garant D et al. (2011). Genetic correlation between resting metabolic rate and exploratory behaviour in deer mice (Peromyscus maniculatus). J Evol Biol 24: 2153-2163.

Forstmeier W (2004). Female resistance to male seduction in zebra finches. Anim Behav 68: 1005-1015.

Forstmeier W (2011). Women have relatively larger brains than men: a comment on the misuse of general linear models in the study of sexual dimorphism. Anat Rec 294: 1856-1863.

Forstmeier W, Martin K, Bolund E, Schielzeth H, Kempenaers B (2011). Female extrapair mating behavior can evolve via indirect selection on males. Proc Natl Acad Sci USA 108: 10608-10613.

Garland T Jr, Carter PA (1994). Evolutionary physiology. Annu Rev Physiol 56: 579-621.

Gebczynski AK, Konarzewski M (2009). Locomotor activity of mice divergently selected for basal metabolic rate: a test of hypotheses on the evolution of endothermy. J Evol Biol 22: $1212-1220$.

Groeneveld E, Kovać M, Mielenz N (2008). VCE User's Guide and Reference Manual Version 6.0. Institue of Farm Animal Genetics, Friedrich Loeffler Institute: Mariensee, Germany.

Houston Al (2010). Evolutionary models of metabolism, behaviour and personality. Philos Trans R Soc Lond Ser B 365: 3969-3975.

Jones AG, Arnold SJ, Bürger R, Houle D (2003). Stability of the G-matrix in a population experiencing pleiotropic mutation, stabilizing selection, and genetic drift. Evolution 57: 1747-1760.

Kane SL, Garland T Jr, Carter PA (2008). Basal metabolic rate of aged mice is affected by random genetic drift but not by selective breeding for high early-age locomotor activity or chronic wheel access. Physiol Biochem Zool 81: 288-300.

Kinghorn B, Kinghorn S. (2010). Pedigree Viewer 64a. Available at. http://wwwpersonal.une.edu.au/ bkinghor/pedigree.htm

Konarzewski M, Książek A (2013). Determinants of intra-specific variation in basal metabolic rate. J Comp Physiol B 183: 27-41.

Lande R, Arnold SJ (1983). The measurement of selection on correlated characters. Evolution 37: 1210-1226.

Lighton JRB (2008). Measuring Metabolic Rates: A Manual for Scientists. Oxford University Press: New York, NY, USA.

McNab BK (1997). On the utility of uniformity in the definition of basal rate of metabolism. Physiol Zool 70: 718-720.

Nespolo RF, Bustamante DM, Bacigalupe LD, Bozinovic F, Houle D (2005). Quantitative genetics of bioenernergetsics and growth-related traits in the wild mammal, Phyllotis dawini. Evolution 59: 1829-1837.

Nilsson J-Å, Åkesson M, Nilsson JF (2009). Heritability of resting metabolic rate in a wild population of blue tits. J Evol Biol 22: 1867-1874.

Phillips PC, Whitlock MC, Fowler K (2001). Inbreeding changes the shape of the genetic covariance matrix in Drosophila melanogaster. Genetics 158: 1137-1145.

Réale D, Garant D, Humphries MM, Bergeron P, Careau V, Montiglio P-O (2010). Personality and the emergence of the pace-of-life syndrome concept at the population level. Phil Trans R Soc Lond Ser B 365: 4051-4063.

Roff D (2000). The evolution of the G matrix: selection or drift? Heredity 84: 135-142.

Rønning B, Jensen H, Moe B, Bech C (2007). Basal metabolic rate: heritability and genetic correlations with morphological traits in the zebra finch. J Evol Biol 20: $1815-1822$.

Schielzeth H (2010). Simple means to improve the interpretability of regression coefficients. Methods Ecol Evol 1: 103-113.

Speakman JR, Król E, Johnson MS (2004). The functional significance of individual variation in basal metabolic rate. Physiol Biochem Zool 77: 900-915.

Tieleman BI, Versteegh MA, Helm B, Dingemanse NJ (2009). Quantitative genetics parameters show partial independent evolutionary potential for body mass and metabolism in stonechats from different populations. J Zool 279: 129-136.

Verhulst S, Holveck MJ, Riebel K (2006). Long-term effects of manipulated natal brood size on metabolic rate in zebra finches. Biol Lett 2: 478-480.

Warton D, Ormerod J (2007). Smatr package version 21. Available at. http://web.maths.unsw.edu. au/ $\sim$ dwarton

White C, Kearney M (2013). Determinants of inter-specific variation in basal metabolic rate. J Comp Physiol B 183: 1-26.

Wolf M, McNamara JM (2012). On the evolution of personalities via frequency-dependent selection. Am Nat 179: 679-692.

Wone B, Sears MW, Labocha MK, Donovan ER, Hayes JP (2009). Genetic variances and covariances of aerobic metabolic rates in laboratory mice. Proc $R$ Soc Ser $B$ 276: 3695-3704. 